\title{
Exploring Physical Properties of Tantalum Carbide at High Pressure and Temperature
}

Zhengang Zhang, ${ }^{\dagger}$ Hao Liang, ${ }^{\ddagger}$ Haihua Chen, ${ }^{*} \dagger, \S$ Juwei Wang, ${ }^{\S}$ Fang Peng, ${ }^{\star}$ and Cheng Lu, ${ }^{*} \|, \perp$

${ }^{\dagger}$ Mechanical Engineering Department of Qinghai University, Xining 810016, P. R. China

${ }^{\S}$ Department of Basic Education, Qinghai University, Xining 810016, P. R. China

${ }^{\ddagger}$ Institute of Atomic and Molecular Physics, Sichuan University, Chengdu 610065, P. R. China

" School of Mathematics and Physics, China University of Geosciences (Wuhan), Wuhan 430074, P. R. China

${ }^{\perp}$ Department of Physics and Astronomy, University of Nevada, Las Vegas, Nevada 89154, United States

*Electronic address: chenghaihua06@163.com

*Electronic address: lucheng@calypso.cn 

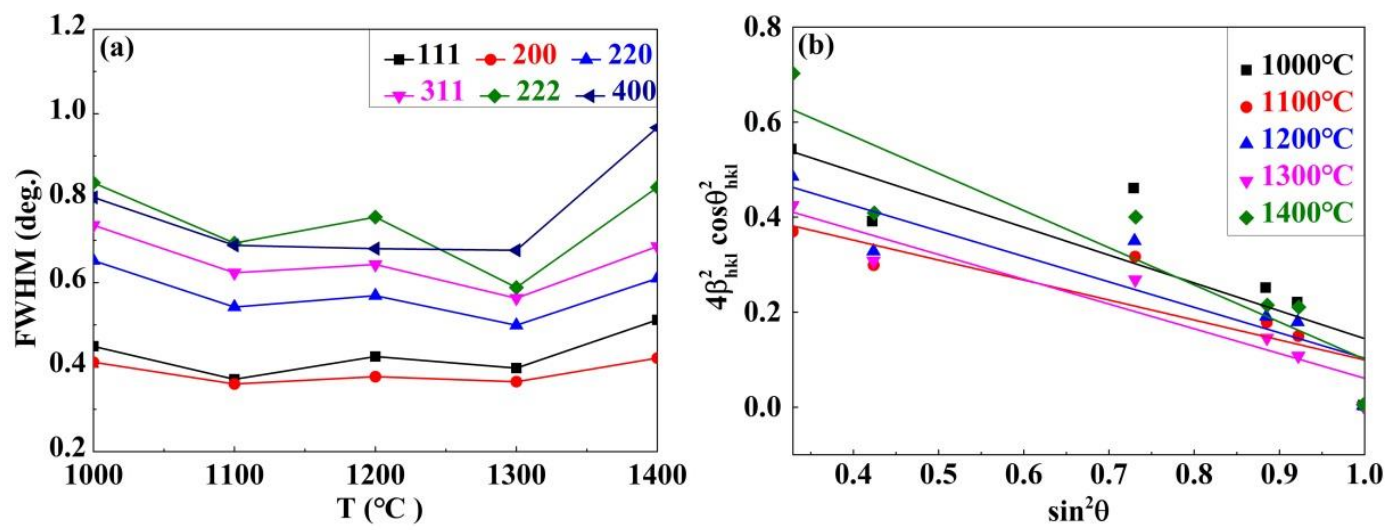

FIG. S1. (a) The FWHM of XRD peaks at (111), (200), (220), (311), (222), (400); (b) $4 \beta_{h k l}^{2} \cos \theta_{h k l}^{2}$ as a function of $\sin ^{2} \theta$. 

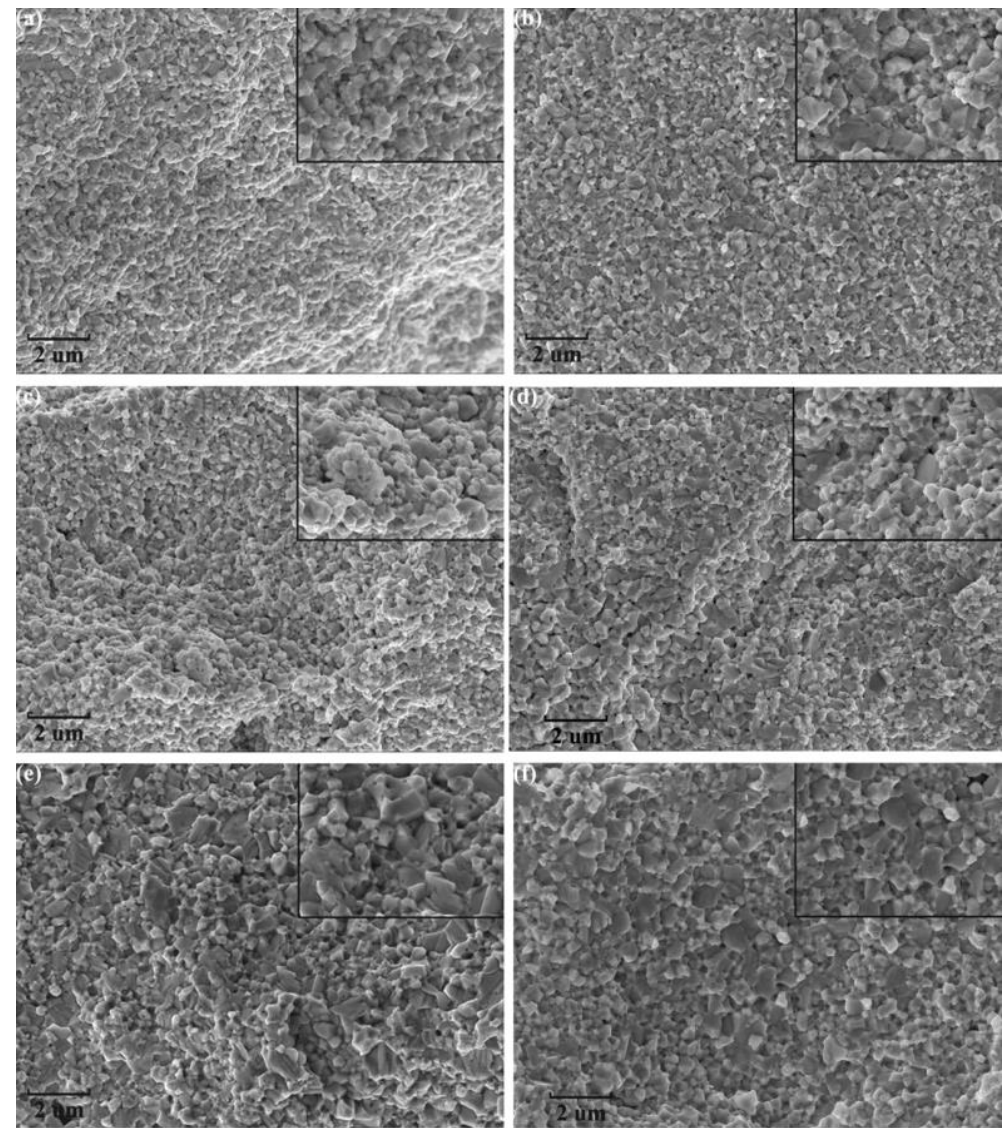

FIG. S2. The SEM of the sintering samples at different temperature. (a) $1000{ }^{\circ} \mathrm{C}$, (b) $1100{ }^{\circ} \mathrm{C}$, (c) $1200{ }^{\circ} \mathrm{C}$, (d) $1300{ }^{\circ} \mathrm{C},(\mathrm{e}) 1400{ }^{\circ} \mathrm{C}$, (f) $1500{ }^{\circ} \mathrm{C}$. 


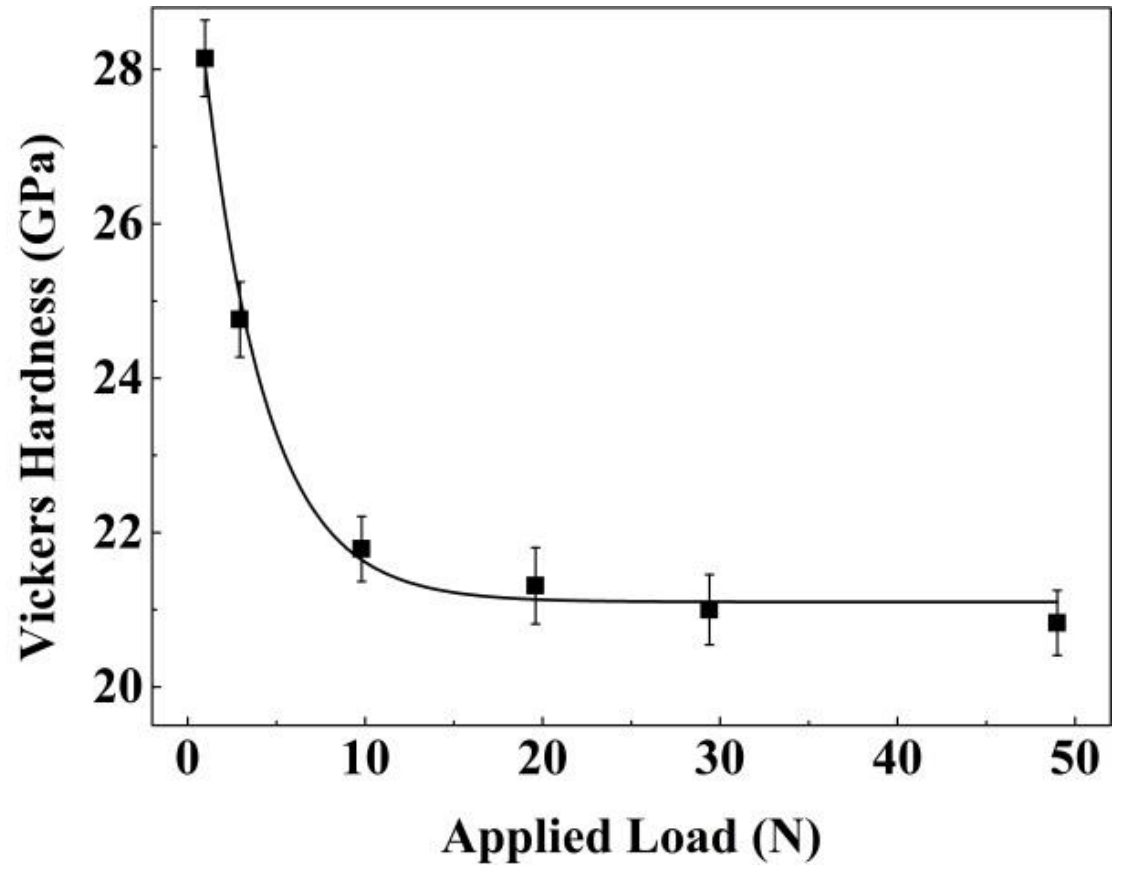

FIG. S3. Vickers hardness of $\mathrm{TaC}$ at $1300{ }^{\circ} \mathrm{C}$ under different loadings. 